\title{
ACOMPARISON OF UNIVERSITY AND SCHOOL TUTORIAL TEACHING
}

\section{Ian Hamilton}

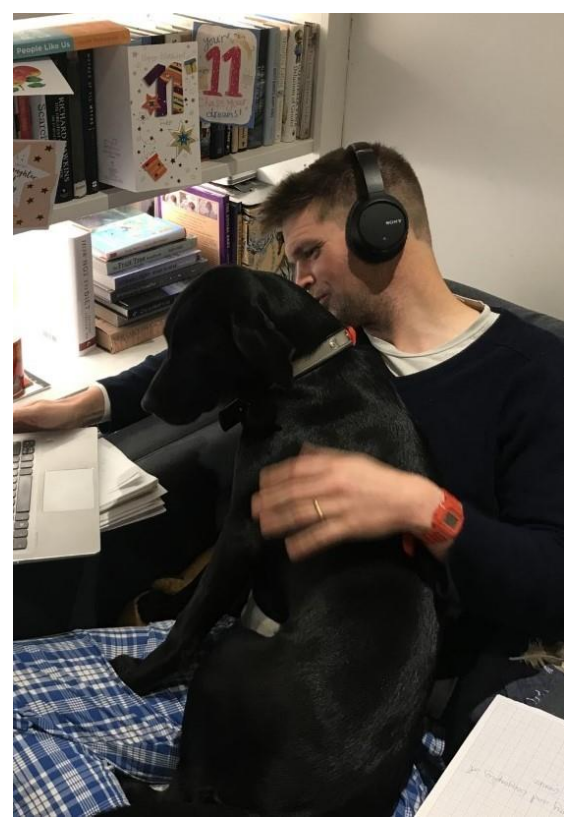

\author{
PhD Student, Statistics, University of Warwick \\ Correspondence: i.hamilton@warwick.ac.uk \\ Webpage: www.warwick.ac.uk/lanHamilton
}

lan is a father of two (8 and 12) with a longstanding interest in teaching. He has experience of education, as a teacher or a parent, in Uganda, Ecuador, Singapore and the UK. Together these experiences have served to reinforce his sense for the value of good teaching. More recently - having slotted a first career as a Foreign Exchange trader in between - he returned to university to study Statistics, where amongst other things, he is looking at some theoretical aspects of the Comparative Judgement methods being increasingly adopted for assessment in educational settings.

\section{Abstract}

In the Spring term of 2021, I performed two online tutorial-based teaching roles. One was with groups of second year university Statistics students; the other was with groups of Year 11 GCSE Mathematics students. In this essay I aim to compare those experiences and draw out some learnings for PGR teaching practice

Keywords: Training, Materials, Community, Engagement, Impact Assessment, Schools

\section{Background}

For some time, I have had an interest in school level teaching. Beyond the self-interest that comes with having children, I have long held an intuition for the compounding effect that good teaching can have and the positive role it can play in society. Over the recent years, it has provided an additional interest to me as an informative example of the opportunities and challenges of taking a more quantitative evidence-based approach to assessing standard practices in an area which, in the past, has relied more on instinct and experience. In the UK, this work has been led by the Educational Endowment Foundation (EEF), formed in 2011 by the Sutton Trust, and funded by the government with the aim of helping to raise the attainment of 3-18 yearolds, particularly those facing disadvantage.

It was through following the EEF that I became aware of the opportunity to be a tutor on the 
National Tutoring Programme (NTP). This programme was set up by the UK government in 2020 to address the shortfall in school education due to the COVID-19 pandemic. The programme was operated through approved tuition partners, and I applied to one of these, called The Brilliant Club (TBC). TBC specialises in taking $\mathrm{PhD}$ students into schools through what they call the Scholar's Programme. In the programme, PhD students design and deliver their own course to inspire school students to apply to a university, focusing on schools where university progression is less common.

Based on academic evidence on the effectiveness of small group teaching, the NTP was delivered mostly to tutorial groups of three students in a course of fifteen one-hour weekly tutorials. I had two of such groups, both based at the same school in another part of the country. As a result, all tutorials were delivered online. In the TBC programme, schools were able to choose four of six modules, each covering a substantial area of the relevant year group curriculum and being covered over three tutorials, with a tutorial at the beginning and end of the course allocated for an initial and final assessment, respectively. The intention was to use these to measure progress. The final tutorial was to be used for feedback and reflection.

At the University, I was allocated two second year Mathematical Statistics tutorial groups with around fifteen students in each group and a one-hour tutorial session for each group, held approximately every other week. As a third year PhD student, this was the sixth term of PGR teaching I had undertaken at a university level.

My intention here is to compare and contrast the experience of delivering the two sets of tutorials and make suggestions based on that. To do this, I will focus on five areas namely, training, materials, community, engagement, and impact assessment. In all cases, I make suggestions for things that could be done to improve the PGR teaching experience.

\section{Training}

TBC required us to complete around three hours of online courses. They were delivered via short videos, often interspersed with exercises. The courses covered three areas namely, general teaching, teaching online, and safeguarding. They were generally very engaging, with interesting and implementable guidance on topics such as questioning techniques and using a backward planning structure to architect the tutorial. Being able to go back to them was also useful. Having said that, I often skipped the intermediate activities. Some parts of these also did not sit well with the teaching of Mathematics or were clearly designed for delivering TBC's mainstay Scholar's Programme rather than the NTP. For example, some of the discussion-promoting strategies were not applicable, and there was some guidance relating to creating material. In addition, we were able to attend live online sessions with the mathematics course content creator going through the material. These live sessions were not mandatory but were very useful for understanding how the material was structured and getting highly relevant tips for its delivery.

\section{Self-reflection was a key practice} emphasised throughout the training and subsequent interactions. This was further encouraged by having a programme officer observe a tutorial and elicit self-reflections, whilst also providing feedback. It was further aided by the requirement to record sessions. While the purpose of this was primarily safeguarding, it acted as a valuable selfevaluation tool. I used it on several occasions to check how I had explained something and determine if I might need to clarify points at the next tutorial.

With regards to university teaching, it is mandatory to do a two and a half hour PGR tutor training session before commencing teaching in my department. This is typically done in the first year of a PhD and no refresher is required, although the same session can be 
voluntarily reattended in subsequent years. In my own case, I do not recall what was covered in that session but have a vague recollection of it being enjoyable and useful. However, by the time I reached my third year, I was basing my practice on what I had done before and found successful. For the advent of online teaching, an additional mandatory training course was required for all tutors. This discussed the practicalities and technologies of delivering online. A tutorial observation was performed in my first year by a teaching fellow, and a 400-word self-reflection essay was required in that first term of teaching. Tutorials are not recorded. A senior tutor is available for half an hour of personal selfreflection over the summer, though this is not mandatory for PGR teachers.

It would not be fair to compare the two training programmes in their entirety given the gap from when I did the university training and my necessarily fuzzier memories. On the one directly comparable part, the online training, I felt TBC placed more emphasis on issues of student interaction online, whereas the university placed more emphasis on the technological alternatives. They were, therefore, usefully complementary. However, I did find the general teaching instruction of the TBC training programme interesting and applicable to both environments. I was able to digest this pedagogic instruction in the context of experience rather than just theory, so I suspect that it had more resonance and meaning for me than it did as pre-experience instruction at the university. One thing that struck me was the very clear focus on the methods and tools by which students could be helped to comprehend the material, for example in the emphasis on the use of visual bar methods. At the university level in contrast, the emphasis in tutorials seems often to be on the quality of answers rather than the route to comprehension, even where the questions are intended to be formative rather than summative. The online format of the TBC instruction was also helpful; being able to engage with it in my own time in smaller portions, was contributory to its impact. In both cases, I found that the materials specifically related to the teaching of my own subject was often the most useful.

Taken together, this might suggest that there is an opportunity to produce more accessible materials specific to the PGR teaching of particular subjects, including in online settings, available to tutors at their discretion. Bitesize training videos introducing relevant pedagogic techniques could provide easy refreshers for PGR tutors seeking to improve their own practice. If there could be an increased emphasis on methods by which students might come to comprehend materials, this may also be beneficial. Since these are challenges faced by PGR tutors at all universities, it would seem to make sense that the production effort here could be shared between departments in the same subject at different universities, so that they can be specific to those subjects while still realising economies of scale. In Mathematics and Statistics, these efforts could, perhaps, be coordinated by groups like the London Mathematical Society, the Institute of Mathematics and its Applications, or the Royal Statistical Society.

The issue of recording is one I will touch on again from the perspective of engagement. In the context of training, I will merely note that it was useful as a selfreflection tool and could be so in the university setting as well.

\section{Materials}

TBC provided us with highly structured materials for all modules at the start of the programme. All materials were produced on PowerPoint documents with a combination of worked examples followed by student practice. It was designed with the idea that a worked example could be stepped through by proceeding through the slides, so that no second device was required. In practice I found it preferable to work with two devices, annotating slides from a tablet, while continuing to appear on screen via another device. I felt this gave a better pacing to how I 
worked through questions and enabled me to visually emphasise key parts from the slide or my annotations to support what I was explaining verbally. While the use of TBC materials for teaching was not mandated, it was strongly encouraged.

In the university setting, the material consisted of a sheet of problems and an accompanying set of worked solutions. This is standard in our department. These were generally released several days before the tutorial which enabled the module tutors to work through the problems, ask questions and spot any mistakes in the solutions provided. Some guidance was given by the module leader as to where to place emphasis, but how the material was delivered was left to the individual tutors.

The material in both settings felt adequate to meeting its target, but there were differences. The university material was fully integrated with the course that the students were following. For example, questions often picked up on worked examples introduced in lectures. TBC materials, while designed from the same national curriculum, bore no relation to the timing of the delivery of those topics in the students' normal school progress.

Students and teachers at the school felt the material was not as helpful as it could be, and the school requested that I teach different topics better integrated with the schedule the school was following. Some of these topics were well-covered in the TBC materials, others less so. This highlighted another feature of the provision of material. It was relatively easy to find good quality material online readily adaptable to teaching particular topics. In particular, it was very easy to find relevant problem sets. This is perhaps to be expected given the large population following the same curriculum and the more defined way in which it is covered. Such a pivot in focus would have been much more difficult in the university environment, but equally would be far less likely to be required. It should also be noted that this was the first year of the NTP and as a result, there had been limited opportunity to hone the material, whereas the Mathematical
Statistics module had existed for several years, and the problem sheet material was not impacted by the move online.

In both settings, the role of the tutorials was very much on doing Mathematics, but it felt that this was a larger part of the school setting overall, whereas the university lecture notes often had a greater focus on the theory. Worked examples and solutions were used without question in the school environment as a fundamental part of the methods and material we were given. The attitude towards worked solutions in the university environment felt much more ambivalent. Indeed, shortly after this teaching experience, I attended a teaching seminar where an academic at a leading Statistics department in the UK explained that the policy of their department was to provide no worked solutions to students. The fear seems to be that students can focus too narrowly on problems of a particular type that they have seen, rather than attaining a broader understanding of the topic through the theory, and that if they know a worked solution will be provided, then they will not do the work themselves. This opinion seems to also be represented in most Statistics textbooks which tend to publish questions without solutions.

In the school setting, there are numerous question banks available, often free, some produced by a single individual or organisation, others effectively crowdsourced. While the challenge of curating such a set of worked solutions would no doubt be much greater than in the common-curriculum school environment, in the age of sophisticated recommender systems, it does not seem impossible that a combination of students and academics could build a navigable question bank for a university Statistics setting. Doing so would make the challenge of questionsetting much easier for academics, while providing a sufficient breadth of questions to students that they can practice and avoid the trap of being focused too much on a single question type. For PGR tutors, it would provide a useful additional resource if students were to request to look at some 
particular topic, or to refer students to if they seem to be struggling in a particular area.

\section{Community}

The NTP recommended to tuition partners that they set up systems by which tutors offered peer support. However, the TBC did not do this. As a result, we set up a WhatsApp group for ourselves. This tended to focus on finding relevant teaching material from what they had provided and mutual reassurance that the low attendance issues that many of us faced were not unique. Beyond this, there was a much wider teaching community that was very stimulating and available in various forms, including blogposts, social media, and podcasts. In particular, I found the Mr Barton Maths podcast thought-provoking and engaging, with topics ranging from the question 'what is learning?' to practical tips on introducing new topics, and informative discussions on educational technology.

With teaching going online at university, a Microsoft Teams channel was set up for each module leader to communicate with the tutors for that module. This created a sense of community that had not been there in previous years. It was used by tutors to report back on how students had done on different problems, errors in worked solutions, or approaches that had worked well or not so well. Often, the benefit was not so much in a tangible change in practice but in the reassurance that your challenges were being shared by others. A wider channel was also set up for all PGR tutors across the department to elicit and provide information, for example to notify of upcoming Teaching Committee meetings and ask for views. Postings often attracted replies and useful conversations ensued. This new sense of community was welcomed. More widely, the department has for a couple of years held Teaching seminars. Before moving online, these were mostly, sometimes exclusively, attended by teaching fellows within the department. It seemed that the move online had also had the effect of encouraging more research fellows who also teach to attend, although they were generally still poorly attended by PGRs.

The increased sense of being part of a team endeavour in providing tutorials was for me, the best part of university teaching in the last year. I believe it would be helpful for the department to be mindful of the benefits of it in the future and look to actively foster and encourage it. For example, departments could make it mandatory for module leaders to host a Teams channel for tutors on their module, where they would be expected to elicit feedback on problem sheets and give guidance as to how they might be delivered, and they could actively seek to promote conversations on a wider PGR tutor chat by asking for opinions on specific things like materials, training or engagement, and by encouraging PGR tutors to attend teaching events across the department.

On a wider level, there seems to be a very weak sense of community across PGR tutors more generally, and PGR tutors are often peripheral to the wider teaching community that does exist in academia. In my own subject area, there was an energetic response amongst academics to the teaching challenges of going online, but the seminars held were, as far as I am aware, almost exclusively focused on issues faced by teaching fellows such as lecture delivery, course structure, mass participation and the like, rather than those of PGRs. There is of course, a challenge here. Often, such communities form around the efforts of particularly enthusiastic and experienced individuals. On the other hand, an experienced PGR tutor is perhaps one with just three years' experience. Establishing the sort of continuity that is required for the building of such communities is, therefore, difficult. This journal itself might be thought of as a welcome effort in that direction, but perhaps other bodies could consider how they might provide the continuity for the enthusiasm of PGRs to be plugged into. This might sit alongside the sort of efforts I described with respect to training. Given the subject-specific challenges of tutoring, it may, as I suggested 
there, be best taken up by relevant crossuniversity bodies.

\section{Engagement}

A key concern in online teaching is the engagement levels of students. There is a concern that the attention of students is difficult to hold through an online portal and there are not the physical cues to assess engagement and understanding that are available when teaching in person.

The NTP setting facilitated very high levels of engagement through the small group sizes - three students when there was full attendance. Most students were either unwilling or unable to use technology that included a camera. However, the small groups meant that questions could be directed to individuals, often with the difficulty of those questions adapted for that individual, and all students demonstrated high engagement throughout the hour of the tutorial, each answering multiple questions. Pleasingly, it was often clear that students had made progress on the material, even within the hour. On the other hand, the tutorials were held immediately after school. They were also scheduled over a period, the second half of which coincided with the students' physical return to school and formal assessments in all subjects that contributed to their final GCSE grades. As such, attendance was poor, with many students presumably opting to spend the time on other studies or just relaxing away from a pressured learning environment. Based on conversations on the tutor WhatsApp group, this poor attendance was found to be a common phenomenon of the NTP.

Engagement here could be seen as very binary. When they attended, engagement was very high, but there was frequently nonattendance, that is, zero engagement.

At the university level, the picture was more mixed. In the Autumn term, I had tutored a module online to first years as a COVIDoverflow to sessions that I was running faceto-face on the same module. There, I had no problem in getting participation including often cameras being left on (when that did not cause streaming difficulties), and questions that I asked were always met with a response. In the Spring term, with a second-year cohort and module, the engagement was notably lower. No one was willing to turn on their camera, and sometimes, questions would elicit no response or simply an "idk" (I don't know) on the chat. It was also noticeable that engagement levels, measured in terms of responses to questions, decreased over the course of the term. Based on observations of the module leader, this seemed to be related to how the students found the material increasingly difficult to keep up with.

A strategy that I like to use in face-toface teaching is to get students to work on problems together in groups (often at whiteboards), before I go through the answer to the question, taking it step by step and coldcalling different individuals at each step. This provides the opportunity for students to attempt a question with support but with the knowledge that they might be asked about any part of it, so it is in their interests to follow what is being discussed in their group, and everyone gets a correct worked solution explained to them. In the first term, it had been possible to somewhat replicate this, even online. The module leader had allocated time to put students into small groups and create their own chats and introduce themselves there. The exercise was designed to help get them familiar with Teams, and for them to meet other students. These groups could then be used to work through problems together, even if this was somewhat less efficient than the in-person non-socially-distanced version. In the second-year group, I tried to do the same, but it was impossible to check if they were being used, and they could be easily disrupted by individuals not attending. My impression was that people reverted to mostly trying to do the questions on their own.

Tentatively, I would suggest that normsetting in an online environment is important. If we want people to have cameras on so we can better judge engagement and 
understanding, then we need to make it clear that this is an expectation from the start of their course, and the message needs to be consistent and repeated. As I mentioned, there also seemed to be a noticeable drop-off in participation over the term. This is a familiar pattern, but online learning would seem to offer a way to ameliorate this. It might be expected that a large proportion of students will struggle to keep up at the end of second term if we consider that, with third term mostly taken up with exams, students are expected to digest most of the taught learning for the year in just the twenty weeks of the first two terms. With the resources all being online, perhaps, module leaders could offer the material early and with a self-directed timetable that allows students to spread the learning over a longer period of time, including holidays. The assignments and quizzes that contributed to marks would still be available only in term time, but learning for students could be better spread. For PGR tutors, this might increase the likelihood of engagement from students as they would be more comfortable with the material, having had a longer time to digest it. While the school terms were no doubt intense, especially for the assessment period, they are longer and the fact that the Year 10 and Year 11 materials were shared suggests that learning over that longer period is one that works better in schools.

Perhaps more controversially, a combination of fees and online learning could be used to incentivise greater tutorial engagement. The online resources created this year, such as recorded lectures, notes, and online assessments, could be offered as part of a lower cost course, stripped of in-person elements such as tutorials and in-person lectures. In this way, it would be made more explicit that students are being charged for inperson elements such as tutorials. It might then be hoped that they would be more invested in making the most of them.

Recording of tutorials may also be thought to have an impact on engagement, potentially both positive and negative. There was no evidence from my school teaching experience that it had a negative impact there, though the smaller groups may have played a role in that. In the university setting, one of the major objections to recording tutorials is that students will be less willing to contribute if a session is recorded. This may be so, though there is no evidence from this particular setting, and there are potential engagement benefits from students being able to go back and review the explanations offered during the tutorial.

\section{Impact Assessment}

The public funding of NTP meant there was an effort to measure the impact of the programme. The idea was that this would be done by having students take an assessment at the start and at the end of the programme, with the results compared to determine progress. The fact that there was no control group seems a significant omission in this design. In practice, a bigger issue was that based on the discussions on the WhatsApp chat, only a small proportion of students completed a start and end of programme assessment, and it is probably not unreasonable to suspect them to be a selfselected conscientious group whose learning was benefiting from being back at school and revising for exams independent of the programme intervention. These metrics were collated for the impact from the four tutors and two subjects that were operated at the school at which I was based and are presumably also being collated at a tuition partner and NTP level. On a qualitative basis, I also received feedback from the programme officer on an observed tutorial.

In our departmental university setting, as far as I am aware, there has never been an attempt to measure the impact or effectiveness of tutorials. We do receive a voluntary feedback survey from students. I received this from only five students across approximately thirty students in the tutorial group. At an aggregate level, there is also the annual National Student Survey. As mentioned in the previous section, perhaps 
this could be improved by mechanisms that meant students valued tutorials more highly. More mundanely, perhaps, mechanisms that provide greater incentives for receiving feedbacks could be put in place. For example, students could be given their module marks at an earlier date if they had completed feedback for that module.

\section{Concluding Remarks}

It is often informative to attempt the same task in two different settings, and it is to be hoped that this was the case here. The observations led to a number of suggestions. Perhaps, the foremost of which is that there would be value in inter-university subject bodies acting as curators to subject PGR teaching practice communities since the transitory nature of the PGR experience means PGRs cannot be expected to reliably do so themselves. Such curation would be expected to consist of the provision of suitable materials, both training and topic-related, and of interactions of PGR teachers to share experiences and best practices. Additional suggestions were made in using the experience of online teaching to allow for alternative scheduling, and more controversially, the offering of lower cost course versions that might increase the value and expectations that students hold for tutorials, thus, incentivising engagement and feedback. It was also suggested that for online teaching, recording might be positive since it increases the usefulness of tutorials to students and assists PGR teachers to improve their practice; or more minimally, that an evaluation of its value would be feasible and helpful. Finally, it is suggested that departments should consider more robust mechanisms to ensure PGR teachers receive good quality feedback.

To cite this article: Ian Hamilton. 2021. "A Comparison of University and School Tutorial Teaching". Journal of PGR Pedagogic Practice, 1, xx-xx. Available at: [url] 